\title{
A Comprehensive Approach Is Vital for Diagnosing COVID-19: A Case of False Negative
}

\author{
Mamtha Balla ${ }^{\mathrm{a}, \mathrm{b}, \mathrm{i}}$, Ganesh Prasad Meruguc ${ }^{\mathrm{c}}$, Mytri Pokal ${ }^{\mathrm{d}}$, Vijay Gayame, \\ Sreedhar Adapa ${ }^{\mathrm{f}}$, Srikanth Naramalag, Venu Madhav Konala ${ }^{\mathrm{h}}$
}

\begin{abstract}
Coronavirus disease 2019 (COVID-19) caused by severe acute respiratory syndrome coronavirus 2 (SARS-CoV-2) is spreading at a rapid pace throughout the world, and the World Health Organization (WHO) declared it as pandemic on March 11, 2020. We present a case of COVID-19 patient whose reverse transcription-polymerase chain reaction (RT-PCR) initially was false negative and later turned positive, which will stress the importance of a comprehensive approach while evaluating a patient with a differential of COVID-19. The clinicians should be aware of the sensitivity and specificities of these tests which can have grave implications on the patient and community if the diagnosis is missed just based on the laboratory tests due to the highly contagious nature of the disease.
\end{abstract}

Keywords: COVID-19; True positives; False negative; RT-PCR; Comprehensive approach; Testing

\section{Introduction}

Coronavirus disease 2019 (COVID-19) became pandemic on March 11, 2020. As of April 17, 2020, there were around $2,263,847$ confirmed cases around the globe and 154,777

Manuscript submitted April 20, 2020, accepted April 29, 2020

aDepartment of Internal Medicine, University of Toledo, Toledo, OH 43606, USA

bPromedica Toledo Hospital, Toledo, OH 43606, USA

'Division of Geriatric Medicine, Department of Family Medicine, University of Toledo, Ohio 43614, USA

${ }^{\mathrm{d} D e p a r t m e n t}$ of Internal Medicine, Navicent Health, Macon, Georgia 31201, USA

eDepartment of Medicine, Interfaith Medical Center, Brooklyn, NY, USA

fDepartment of Internal Medicine, Division of Nephrology, Adventist Medical Center, Hanford, CA 93230, USA

gDepartment of Internal Medicine, Division of Rheumatology, Adventist Medical Center, Hanford, CA 93230, USA

hDepartment of Internal Medicine, Division of Medical Oncology, Ashland Bellefonte Cancer Center, Ashland, KY 41169, USA

${ }^{i}$ Corresponding Author: Mamtha Balla, Department of Internal Medicine, University of Toledo, Toledo, OH 43606, USA. Email: athamam@gmail.com

doi: https://doi.org/10.14740/jocmr4173 confirmed deaths, all 185 countries, areas, or territories with cases [1]. Moreover, the USA has 708,622 cases [1]. Pandemic COVID-19 is a war between humans and virus. Only with a multidirectional approach, we can temporarily mitigate this problem by taking community measures until we have a permanent solution in the form of a vaccine, which is efficacious, or medication to treat severe acute respiratory syndrome coronavirus 2 (SARS-CoV-2)/COVID-19. As per the World Health Organization (WHO) tracing, isolation, and testing are backbones of COVID-19 response [2]. Improving the efficiency of diagnosing suspected cases and isolating is the only way we can decrease the burden of the pandemic in the community. The sensitivity of reverse transcription-polymerase chain reaction (RT-PCR) depends upon the person performing, the timing of the test, the site of the testing, low viral load, improper sampling techniques, and mutations in the viral genome [3, 4]. So, the clinician needs to understand the implications of negative testing; therefore, it is necessary to utilize a comprehensive approach to diagnose a patient. False-negative testing will not only falsely assure patients but also have grave implications on public health due to its highly contagious nature. Every clinician should adopt a comprehensive and multi-dimensional approach to diagnose COVID-19.

\section{Case Report}

A 40-year-old woman presented with a chief complaint of productive cough, shortness of breath, and wheezing for a week with symptoms getting worse over 3 days. The patient denied any fever, nausea, vomiting, or diarrhea. She denied any sick contacts or recent travel history. Her past medical history was significant for hypertension noncompliant with her medications, type 2 diabetes mellitus, and obstructive sleep apnea on continuous positive airway pressure (CPAP) at night. She had no history of smoking or drug use. The patient was currently taking metformin $500 \mathrm{mg}$ one tablet daily with breakfast.

Initial vital signs on presentation showed a temperature of blood pressure of 137/89 mm Hg, pulse rate of $77 \mathrm{bpm}$, respiratory rate of 22 breaths per minute, and oxygen saturation of $97 \%$ on $3 \mathrm{~L}$ of the nasal cannula. A review of systems was positive for cough and shortness of breath. The patient was not in acute distress and examination of the head, eyes, ears, nose, and throat (HEENT) was normal, she had normal rate 
Table 1. Summary of Laboratory Abnormalities

\begin{tabular}{ll}
\hline Laboratory findings & Patients value (normal values) \\
\hline White blood cell count & $8.0\left(4.8-10.8 \times 10^{9} / \mathrm{L}\right)$ \\
Absolute lymphocyte count & $1.5\left(1.0-3.5 \times 10^{9} / \mathrm{L}\right)$ \\
Procalcitonin & $<0.05(<0.05)$ \\
D-dimer & $186(<255 \mathrm{ng} / \mathrm{mL})$ \\
CRP (high) & $13.2(0.000-0.744 \mathrm{mg} / \mathrm{dL})$ \\
LDH (high) & $390(100-235 \mathrm{U} / \mathrm{L})$ \\
Serum ferritin & $284(11-307 \mathrm{ng} / \mathrm{mL})$ \\
Erythrocyte sedimentation rate (high) & $59(0-20 \mathrm{~mm} / \mathrm{h})$ \\
Respiratory viral panel & Negative \\
Vitamin D (low) & $17(>30)$ \\
INR (high) & $1.3(0.9-1.2)$ \\
BMI & $42.91 \mathrm{~kg} / \mathrm{m}^{2}$ \\
\hline
\end{tabular}

CRP: C-reactive protein; LDH: lactate dehydrogenase; INR: international normalized ratio; BMI: body mass index.

and regular rhythm with no additional sounds on heart examination, patient's respiratory effort was normal, no respiratory distress was noted, and she was noted to have diminished bilateral breath sounds. Abdominal, skin, neurological examination was benign. No positive findings were noted.

The initial laboratory evaluation was summarized in Table 1. Considering active COVID-19 pandemic and high suspicious for COVID-19, emergency room (ER) physician opted for chest computed tomography (CT) directly to decrease cross contamination and exposure to technicians. Chest CT showed ground-glass opacity and bilateral bases suggestive of viral pneumonia, as shown in Figure 1.

Due to current pandemic nature patient was subsequently admitted for SARS-CoV-2/COVID-19 rule out and the patient was placed in airborne precautions. The patient was started on empirical treatment with azithromycin along with other medications including zinc sulfate $220 \mathrm{mg}$ once a day, vitamin $\mathrm{C}$ $1,000 \mathrm{mg}$ once a day, probiotics one tablet once a day, melatonin $3 \mathrm{mg}$ at night as an adjunctive medication [5-7] for COVID-19, vitamin D 2,000 mg once daily for vitamin D deficiency, enoxaparin $40 \mathrm{mg}$ subcutaneous daily as deep vein thrombosis prophylaxis and mucinex as symptomatic management for cough.

Nasopharyngeal swab for RT-PCR was sent for SARS-
CoV-2/COVID-19 testing. On day 3 of the hospitalization, patient's symptoms started getting better. On day 3 of the hospitalization test, RT-PCR for SARS-CoV-2/COVID-19 from nasopharyngeal swab came back negative. As the patient's initial symptoms, the requirement of oxygen, and laboratory findings (lymphopenia, elevated lactate dehydrogenase (LDH), Creactive protein (CRP), erythrocyte sedimentation rate (ESR) and negative respiratory virus panel) with comorbid conditions including hypertension, morbid obesity, type 2 diabetes and radiological findings were suspicious for COVID-19, to get more help infectious disease specialist was consulted. The patient has five kids living with her; in order to discharge her safely so that her family and community will be protected, we opted to get second testing due to strong radiological findings including bilateral ground-glass opacities in bases, clinical signs, symptoms and comorbid conditions. Infectious disease specialist classified the patient as the medium risk for the presence of COVID-19 disease based on imaging alone as well. The second test from nasopharyngeal swab RT-PCR was sent on the day 3 of her hospitalization. The patient was gradually weaned off her oxygen, and it was felt that she has a low risk for progression to severe disease. On day 4 of her hospitalization, the results of the second RT-PCR test were reported positive. The patient's complete hospital course was also shown in

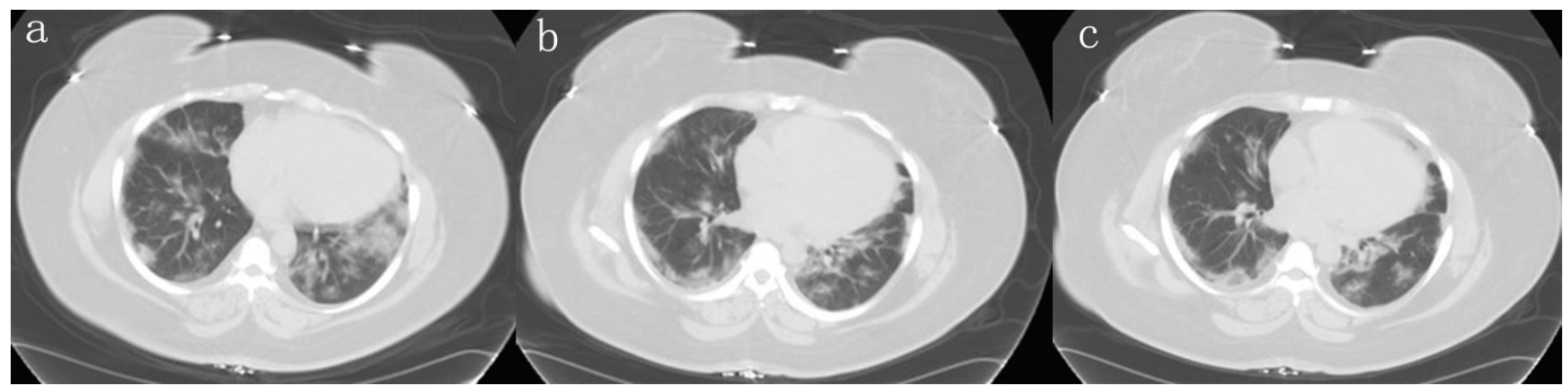

Figure 1. Different planes of the CT scan showing bilateral worsening of ground-glass opacities at bases. CT: computed tomography. 


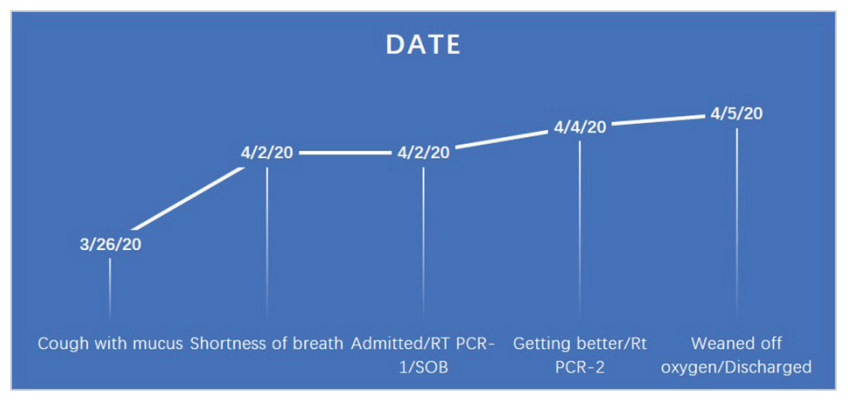

Figure 2. Time course of the patient since the symptoms started until recovered and discharged.

Figure 2. The patient was discharged with a recommendation for strict home isolation for 2 weeks.

\section{Discussion}

Testing, isolation, and tracing are the backbone for controlling the COVID-19 pandemic, so every effort should be made to appropriately diagnose suspected patients and minimizing false negative is essential to prevent the spread of the disease. Since RT-PCR is a gold standard method to identify and confirm COVID-19 [8], a false-negative result, can hamper the control of the pandemic. Due to its high contagious nature, it will not only have an effect on a personal level but also at a community level. False-negative RT-PCR for COVID-19 case reports are reports from several cities of China [8, 9], but none have been reported so far from Western countries, including the USA. We are reporting a case of false-negative COVID-19, which was tested again due to the highly suspicious nature of the diagnostic tests, which turned to true positive. As per West et al, even if a test has $90 \%$ sensitivity, as per the example given in California, COVID-19 might have exceeded $50 \%$ by mid-May 2020 , and even if $1 \%$ of the population were tested, a total of 20,000 false-negative results would be expected [10]. Also, in the same article, if COVID-19 infection among healthcare workers (greater than four million) providing direct patient care was even $10 \%$, more than 40,000 false-negative results are expected if everyone gets tested [10]. According to Li et al, RT-PCR has $20 \%$ of the false-negative rate. So, clinical features, laboratory test results as well as $\mathrm{CT}$ features of the patient help in identifying and diagnosing patients [8]. So, a multi-prong approach is essential in the diagnosis of COVID-19. According to the research, 96\% of the COVID-19 patients present with multiple bilateral and peripheral consolidation and ground-glass opacities in the chest CT $[11,12]$. Chest CT abnormalities are very vital in diagnosing COVID-19. When the COVID-19 pandemic was severe in China, the National Health Commis-

Table 2. Different Methods of Testing

\begin{tabular}{llc} 
Methods of testing & What the test interprets & Turnaround time \\
\hline Neutralization assay & $\begin{array}{l}\text { Tests to look for active antibodies in subject serum which can inhibit virus } \\
\text { growth ex vivo. Indicates if the patient is protected against future infection. }\end{array}$ & $3-5$ days \\
ELISA & $\begin{array}{l}\text { Quantify the presence or absence of antibodies } \\
\text { against the virus in the subject's serum. }\end{array}$ & $1-5 \mathrm{~h}$ \\
RDT & $\begin{array}{l}\text { Qualitatively tests for the presence or absence of antibodies against } \\
\text { virus in the subject's serum. Cannot quantify the antibody titer. }\end{array}$ & $10-30$ min \\
\hline
\end{tabular}

ELISA: enzyme-linked immunosorbent assay; RDT: rapid diagnostic test.

Table 3. Diagnostic Tests Approved for COVID-19 in the USA as per FDA

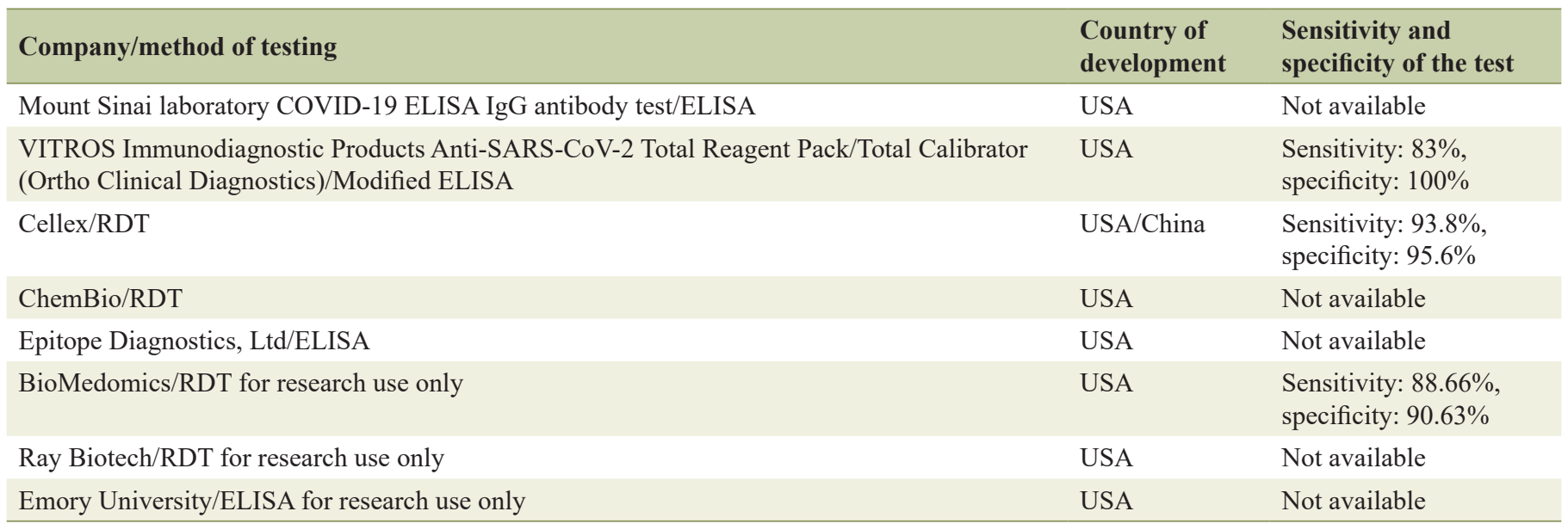

ELISA: enzyme-linked immunosorbent assay; COVID-19: coronavirus disease 2019; FDA: Food and Drug Administration; IgG: immunoglobulin G; RDT: rapid diagnostic test. 
sion of China amended criteria to diagnose in Hubei province with "clinical diagnosis" to include patients with pneumonia in their chest CT irrespective of their RT-PCR COVID-19 results [13]. Different methods of testing, as well as diagnostic tests approved for COVID-19 in the USA as per the Food and Drug Administration (FDA), are summarized in Tables 2 and 3 , respectively $[11,14]$. There is a recent report from Cleveland clinic published in National Public Radio (NPR), after testing 239 specimens, known to contain coronavirus using five of the most commonly used methods for coronavirus testing. Abbott ID NOW was able to detect the virus in about $85 \%$ of the samples approximately as per the study. Another test used in the study, DiaSorin Simplexa, detected only $89.3 \%$ of infections. Therefore, Cleveland clinic is currently using a test developed by the Centers for Disease Control (CDC), which detected $100 \%$ positive samples and another test made by Roche, which detected $96.5 \%$ of samples, as well as a test made by Cepheid, detected $98.2 \%$ of infected samples. As per statement from Abbott, they mentioned that study used viral transport media and errors might happen with viral transport media and it has communicated to healthcare facilities advising them to test directly with the swab, to yield reliable results [15]. This is Abbott's response to the study from Cleveland clinic - the use viral transport media to test the samples, however, the results would be affected if they are not directly from the swab. There are other factors, such as inadequate sampling, as testing requires the collection of secretions in the nasopharynx and rotating the swab several times. The test is not an easy procedure to perform or for patients to tolerate [16]. Other factors such as laboratory techniques, the medium of transportation, the timing of the testing, and faulty testing kits can also influence the test results.

\section{Conclusions}

In conclusion, we reported a false-negative result of RT-PCR for COVID-19/SARS-CoV-2 infection based on a multidirectional approach; early and accurate diagnosis of COVID-19 is essential in dealing with pandemic effectively. False-negative results can have a significant implications on public health due to high reproductive factor of COVID-19.

\section{Acknowledgments}

I thank all the authors who contributed to case report.

\section{Financial Disclosure}

None to declare.

\section{Conflict of Interest}

None to declare.

\section{Informed Consent}

It was obtained from the patients.

\section{Author Contributions}

MB collected and reviewed patients' chart; GPM, MP, VG, $\mathrm{SA}, \mathrm{SN}$, and VMK contributed in writing Introduction, Discussion and Conclusion. All authors contributed equally to preparation of this manuscript and all of the authors reviewed the manuscript and agreed with the findings and interpretation.

\section{Data Availability}

The authors declare that data supporting the findings of this study are available within the article.

\section{Abbreviations}

RT-PCR: reverse transcription-polymerase chain reaction; COVID-19: coronavirus disease 2019; SARS-CoV-2: severe acute respiratory syndrome coronavirus 2; WHO: World Health Organization; CPAP: continuous positive airway pressure; HEENT: head, eyes, ears, nose, and throat; ER: emergency room; CT: computed tomography; LDH: lactate dehydrogenase; CRP: C-reactive protein; ESR: erythrocyte sedimentation rate; FDA: Food and Drug Administration; CDC: Centers for Disease Control; INR: international normalized ratio; BMI: body mass index; IgG: immunoglobulin G; NPR: National Public Radio

\section{References}

1. John Hopkins University in medicine coronavirus resource Center. Available from https://coronavirus.jhu. edu/map.html (Accessed 18 April 2020).

2. World Economic Forum - Global Agenda. https://www. weforum.org/agenda/2020/03/testing-tracing-backbonewho-coronavirus-wednesdays-briefing.

3. Ai T, Yang Z, Hou H, Zhan C, Chen C, Lv W, Tao Q, et al. Correlation of chest CT and RT-PCR testing in coronavirus disease 2019 (COVID-19) in China: a report of 1014 cases. Radiology. 2020:200642.

4. Winichakoon P, Chaiwarith R, Liwsrisakun C, Salee P, Goonna A, Limsukon A, Kaewpoowat Q. Negative nasopharyngeal and oropharyngeal swabs do not rule out COVID-19. J Clin Microbiol. 2020;58(5):e00297-20.

5. Zhang L, Liu Y. Potential interventions for novel coronavirus in China: A systematic review. J Med Virol. 2020;92(5):479-490.

6. Gao QY, Chen YX, Fang JY. 2019 Novel coronavirus infection and gastrointestinal tract. J Dig Dis. 2020;21(3):125-126.

7. Zhang R, Wang X, Ni L, Di X, Ma B, Niu S, Liu C, et al. 
COVID-19: Melatonin as a potential adjuvant treatment. Life Sci. 2020;250:117583.

8. Li D, Wang D, Dong J, Wang N, Huang H, Xu H, Xia C. False-negative results of real-time reverse-transcriptase polymerase chain reaction for severe acute respiratory syndrome coronavirus 2: role of deep-learning-based CT diagnosis and insights from two cases. Korean J Radiol. 2020;21(4):505-508.

9. Chen Z, Li Y, Wu B, Hou Y, Bao J, Deng X. A patient with COVID-19 presenting a false-negative reverse transcriptase polymerase chain reaction result. Korean J Radiol. 2020;21(5):623-624.

10. West CP, Montori VM, Sampathkumar P. COVID-19 testing: the threat of false-negative results. In Mayo Clinic Proceedings. Elsevier. 2020.

11. Chung M, Bernheim A, Mei X, Zhang N, Huang M, Zeng $\mathrm{X}$, Cui J, et al. CT imaging features of 2019 novel coronavirus (2019-nCoV). Radiology. 2020;295(1):202-207.
12. Xie X, Zhong Z, Zhao W, Zheng C, Wang F, Liu J. Chest CT for typical 2019-nCoV pneumonia: relationship to negative RT-PCR testing. Radiology. 2020:200343.

13. China N.H. Diagnosis and treatment protocols of pneumonia caused by a novel coronavirus (trial version 5). Beijing: National Health Commission of the People's Republic of China. 2020.

14. John Hopkins center for health security. Serology-based tests for COVID-19. https://www.centerforhealthsecurity. org/resources/COVID-19/serology/Serology-based-testsfor-COVID-19.html.

15. https://www.npr.org/sections/health-shots/2020/04/21/ $838794281 /$ study-raises-questions-about-false-negatives-from-quick-covid-19-test.

16. Carver C, Jones N. Comparative accuracy of oropharyngeal and nasopharyngeal swabs for diagnosis of COVID-19. Oxford COVID-19 evidence service team centre for evidence based medicine. 2020. 